\title{
Left ventricular dysfunction after mitral valve repair-the fallacy of "normal" preoperative myocardial function
}

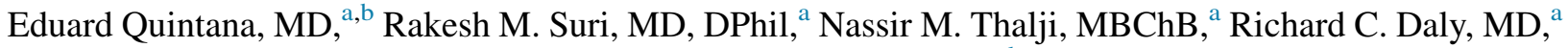 \\ Joseph A. Dearani, MD, ${ }^{a}$ Harold M. Burkhart, MD, ${ }^{a}$ Zhuo Li, MSc, ${ }^{d}$ Maurice Enriquez-Sarano, MD, ${ }^{c}$ and \\ Hartzell V. Schaff, MD
}

Objective: A proportion of patients experience a decrease in left ventricular (LV) ejection fraction (EF) after mitral valve repair; however, predictors and long-term consequences remain unclear.

\begin{abstract}
Methods: A study of 1705 patients with severe, degenerative mitral valve regurgitation and normal preoperative EF ( $>60 \%$ ) undergoing mitral valve repair from 1993 to 2012 was performed. Multivariate logistic regression and Cox proportional hazards models were used to determine the predictors of early postoperative LV dysfunction $(\mathrm{EF}<50 \%)$ and long-term survival, respectively.
\end{abstract}

\begin{abstract}
Results: Postoperative outcomes were comparable between patients; however, those with an EF of $<50 \%$ ( $n=314$, $18.4 \%$ ) had significantly greater enlargement in systolic dimension (left ventricular end-systolic diameter, $-0.6 \mathrm{vs}$ $4.3 \mathrm{~mm} ; P<.001)$ and decrease in right ventricular systolic pressure $(-2.7 \mathrm{vs}-7.8 \mathrm{~mm} \mathrm{Hg} ; P<.001)$ immediately after repair. On longitudinal follow-up, early LV impairment persisted, with $\mathrm{EF}$ recovering to preoperative levels $(>60 \%)$ in only one third of patients with postrepair EF $<50 \%$ versus two thirds of those with an EF of $\geq 50 \%$ $(P<.001)$. The overall survival at 5,10 , and 15 years of follow-up was $95 \%, 85 \%$, and $70.8 \%$, respectively. Although early postoperative $\mathrm{EF}<50 \%$ was not a significant determinant of late survival, when adjusting for older age (hazard ratio [HR], 1.09), hypertension (HR, 1.38), New York Heart Association class III or IV (HR, 1.71), and preoperative atrial fibrillation (HR, 2.33), postoperative $\mathrm{EF}<40 \%$ conferred a $70 \%$ increase in the hazard of late death (HR, 1.74; $95 \%$ confidence interval, $1.03-2.92 ; P=.037)$. A preoperative right ventricular systolic pressure $>49 \mathrm{~mm} \mathrm{Hg}$ and left ventricular end-systolic diameter $>36 \mathrm{~mm}$ were independently associated with a 4.4- and 6.5-fold increased risk of developing a postoperative $\mathrm{EF}<40 \%(P<.001$, for both $)$.

Conclusions: De novo postoperative LV dysfunction is not uncommon in patients with "normal" preoperative EF undergoing mitral valve repair. LV dysfunction can persist, impairing recovery of LV size, function, and survival. The consideration of mitral repair before the onset of excessive LV dilation or pulmonary hypertension, even in those with preserved EF, seems warranted. (J Thorac Cardiovasc Surg 2014;148:2752-62)
\end{abstract}

Although mitral valve repair is the only safe and effective method of treating patients with severe degenerative mitral

From the Divisions of Cardiovascular Surgery, ${ }^{\text {a }}$ Cardiovascular Diseases, and Biomedical Statistics and Informatics, ${ }^{\mathrm{d}}$ Mayo Clinic College of Medicine, Rochester, Minn; and Universitat de Barcelona, ${ }^{\mathrm{b}}$ Barcelona, Spain.

Disclosures: The Division of Cardiovascular Surgery, Mayo Clinic, reports research relationships with Edwards Lifesciences, Medtronic, and St Jude Medical. Dr Suri is the national principal investigator for and reports patent applications from the Food and Drug Administration trial of the Sorin PERCEVAL valve; a member of the steering committee of the St Jude PORTICO trial; a member of the clinical selection committee and co-principle investigator for the Abbott COAPT trial; site principal investigator for the Edwards PARTNER II trial; and reports grant support from Edwards, Abbott, and St Jude. Dr Daly reports consulting fees from and equity ownership in Neochord Inc. All other authors have nothing to disclose with regard to commercial support.

E.Q., R.M.S., and N.M.T. contributed equally to this article.

Read at the 94th Annual Meeting of The American Association for Thoracic Surgery, Toronto, Ontario, Canada, April 26-30, 2014.

Received for publication April 28, 2014; revisions received June 26, 2014; accepted for publication July 5, 2014; available ahead of print Aug 28, 2014.

Address for reprints: Rakesh M. Suri, MD, DPhil, Division of Cardiovascular Surgery, Mayo Clinic College of Medicine, 200 First St SW, Rochester, MN 55905 (E-mail: suri.rakesh@mayo.edu).

$0022-5223 / \$ 36.00$

Copyright (c) 2014 by The American Association for Thoracic Surgery

http://dx.doi.org/10.1016/j.jtcvs.2014.07.029 valve regurgitation (MR), an international debate persists regarding the need for, and timing of, "early" surgical intervention. ${ }^{1-3}$ According to the latest American College of Cardiology/American Heart Association guidelines, ${ }^{4}$ in the absence of class I indications for surgical correction, asymptomatic patients with normal left ventricular (LV) function (ejection fraction $[\mathrm{EF}]>60 \%$ and $\mathrm{LV}$ endsystolic diameter [LVESD] $\geq 40 \mathrm{~mm}$ ) are only offered early repair as a class IIa recommendation.

Because LV dysfunction assessed by echocardiography is often underestimated in patients with severe $\mathrm{MR},{ }^{5}$ reliance on EF alone can be unreliable and delay referral for surgical correction. Even in the presence of "normal" LV function, chronic volume overload caused by severe MR can lead to adverse consequences, such as atrial fibrillation, pulmonary hypertension, and early postoperative LV dysfunction-all of which can affect long-term survival. ${ }^{6,7}$ While the aim of "early surgery" is to prevent these sequelae and improve patient prognosis, the predictors of both latent LV dysfunction and the onset of guideline-based triggers in patients with "normal" preoperative LV function remain unclear. 


$$
\begin{aligned}
& \text { Abbreviations and Acronyms } \\
& \begin{aligned}
\text { CI } & =\text { confidence interval } \\
\mathrm{EF} & =\text { ejection fraction } \\
\mathrm{HR} & =\text { hazard ratio } \\
\mathrm{LV} & =\text { left ventricular } \\
\mathrm{LVESD} & =\mathrm{LV} \text { end-systolic diameter } \\
\mathrm{MR} & =\text { mitral valve regurgitation } \\
\mathrm{OR} & =\text { odds ratio } \\
\mathrm{RVSP} & =\text { right ventricular systolic pressure }
\end{aligned}
\end{aligned}
$$

The ability to ascertain the ideal timing for mitral valve repair to optimize survival and minimize morbidity pivots on the a priori assumption that, in the absence of symptoms, both the extent to which LVEF will be altered early after mitral valve repair surgery ${ }^{8}$ and the exact subset of patients with "normal" EF who are at risk of developing severe LV dysfunction is predictable on an individual basis. ${ }^{9}$ A recent report $^{10}$ suggested that preoperative pulmonary hypertension, atrial fibrillation, and increased LVESD, ${ }^{10}$ all predicted an increased risk of postoperative LV dysfunction after mitral valve repair. However, it is important to note that this and other populations studied to date have included patients with evidence of preoperative LV dysfunction (classically LVEF $<50 \%)^{11}$ before surgery. Whether these findings are relevant in those with "normal" preoperative $\mathrm{EF}$ is unknown. Because of this knowledge gap, our intent was to study a population of patients with echocardiographically confirmed "normal" LVEF before isolated degenerative mitral valve repair in the absence of ischemic disease to (1) elucidate the frequency and predictors of an early decline in LV systolic function, (2) analyze the timing and extent of reverse LV remodeling, and (3) delineate a quantitative threshold for early postoperative LVEF that might influence long term survival.

\section{METHODS}

\section{Study Subjects}

The Mayo Clinic institutional review board approved the present study. From January 1, 1993 to June 30, 2012, 5258 patients underwent conventional open or minimally invasive mitral valve repair for degenerative MR (ie, myxomatous disease or fibroelastic deficiency) at Mayo Clinic Rochester. Our investigation was limited to patients with preoperative echocardiographic evidence of normal LVEF $(>60 \%)$. Those with concomitant tricuspid valve repair, patent foramen ovale closure, or surgery for atrial fibrillation were eligible for inclusion. The exclusion criteria were concomitant coronary artery bypass grafting, a history of myocardial infarction or coronary disease (epicardial coronary lesions $>50 \%$ ), previous mitral valve intervention, active endocarditis, mitral stenosis, congenital heart disease, cardiomyopathy, or refusal of patient consent. A total of 1705 patients met the enrollment criteria and were analyzed in the present study.

The patients were stratified according to the presence or absence of early postoperative LV dysfunction, defined as an LVEF $<50 \%$ on predismissal echocardiographic assessment. ${ }^{10,12}$

\section{Data Collection}

Patient demographics, medical and surgical history, baseline cardiac status, and perioperative data were derived from the Mayo Clinic's Division of Cardiovascular Surgery's electronic patient database and by manual review of the patient medical records. Follow-up data and vital status were obtained from review of the medical records from our institution and outside centers, formal health assessment questionnaires sent to patients and next-of-kin, and the Social Security Death Index.

\section{Echocardiographic Analysis}

All patients underwent transthoracic echocardiographic evaluation before and after mitral valve repair surgery. Echocardiographic imaging was performed at a median of 20 days preoperatively and 4 days postoperatively. Postoperative echocardiography is performed routinely before dismissal at our institution to re-evaluate valve and cardiac function and to rule out postoperative complications. At postoperative echocardiography, the patients were not receiving inotropic support.

Echocardiographic studies were performed according to routine clinical practice. Two-dimensional direct measurements from parasternal long-axis views or M-mode echocardiography were used to obtain LV measurements. EF was obtained using differences in ventricular dimensions. The postoperative degree of MR was assessed semiquantitatively using scales ranging from 1 to 4 by Doppler echocardiography dictated by the American Society of Echocardiography. ${ }^{13}$ A diagnosis of flail segment was made based on failure of leaflet coaptation, with rapid systolic movement of the affected leaflet tips toward the left atrium. Patients with features consistent with other diseases such as ischemic mitral regurgitation, functional, or nonprolapsing segments were excluded.

We analyzed the long-term postoperative changes in several key variables, including the LVEF, LV diameters (LV end-diastolic diameter, LVESD), and left atrial size, within the groups of patients with a postoperative LVEF of $<50 \%$ and LVEF of $\geq 50 \%$ and compared these changes between the 2 groups.

\section{Surgical Procedure}

Mitral valve repair was performed through a partial or full sternotomy or using a minimally invasive approach (ie, port access/right lateral thoracotomy or robot-assisted technique). Valve repair was performed with a variety of surgical techniques used at Mayo Clinic. For posterior leaflet prolapse, triangular leaflet resection is typically performed. Anterior leaflet prolapse is commonly corrected using Gore-Tex artificial neochordal replacement (expanded polytetrafluoroethylene, Gore-Tex; W.L. Gore \& Associates, Inc, Flagstaff, Ariz). Mitral valve annuloplasty is typically performed using a standard length flexible posterior band $(63 \mathrm{~mm})$ anchored with interrupted Ethibond stitches between the left and right fibrous trigones. ${ }^{14-16}$

\section{Statistical Analysis}

Categorical variables are reported as the frequency and percentage and continuous variables as the mean \pm standard deviation or median and interquartile range, as appropriate. Categorical variables were compared between patients with and without an early postoperative LVEF of $<50 \%$ using the chi-square test or Fisher's exact test. Continuous variables were compared using Student's $t$ test or the Wilcoxon rank sum test, as appropriate.

Logistic regression models were used to determine the predictors of early postoperative LV impairment. Kaplan-Meier methods were used to estimate survival after mitral valve repair, with patients censored at last known follow-up. Cox proportional hazards models were used to identify univariate and multivariate predictors of long-term all-cause mortality. Multivariate Cox models constructed using significant variables from univariate analysis $(P<.05)$ corresponded to models derived by backward-and-forward stepwise methods. To examine the relationship between postoperative LVEF as a continuous parameter and the hazard of late death, we used penalized smoothing splines. ${ }^{17}$ Finally, we identified 
TABLE 1. Baseline characteristics

\begin{tabular}{|c|c|c|c|c|}
\hline \multirow[b]{2}{*}{ Variable } & \multicolumn{3}{|c|}{ Predismissal LVEF } & \multirow[b]{2}{*}{$P$ valu } \\
\hline & All patients $(n=1705)$ & $\geq \mathbf{5 0} \%(\mathbf{n}=\mathbf{1 3 9 1})$ & $<\mathbf{5 0} \%(\mathbf{n}=\mathbf{3 1 4})$ & \\
\hline \multicolumn{5}{|l|}{ Clinical characteristics } \\
\hline Age (y) & $58.8 \pm 13.1$ & $59 \pm 12.8$ & $57.7 \pm 14$ & .18 \\
\hline Atrial fibrillation & $105(6.2)$ & $80(5.7)$ & $25(7.9)$ & .14 \\
\hline BMI $\left(\mathrm{kg} / \mathrm{m}^{2}\right)$ & $25.9 \pm 4.3$ & $26 \pm 4.2$ & $25.5 \pm 4.6$ & .002 \\
\hline $\operatorname{BSA}\left(\mathrm{m}^{2}\right)$ & $1.93 \pm 0.23$ & $1.93 \pm 0.23$ & $1.94 \pm 0.24$ & .95 \\
\hline COPD & $108(6.3)$ & $84(6.1)$ & $24(7.7)$ & .29 \\
\hline Diabetes & $45(2.6)$ & $38(2.7)$ & $7(2.2)$ & .61 \\
\hline Female sex & $575(34)$ & $487(35)$ & $88(28)$ & .018 \\
\hline Hypertension & $693(40.7)$ & $589(42.4)$ & $104(33.2)$ & .003 \\
\hline NYHA class III or IV & $496(29.2)$ & $393(26.3)$ & $103(32.9)$ & .11 \\
\hline Peripheral vascular disease & $68(3.9)$ & $52(3.7)$ & $14(4.4)$ & .54 \\
\hline Renal failure (creatinine $>1.4 \mathrm{mg} / \mathrm{dL}$ ) & $10(0.9)$ & $8(0.9)$ & $2(1)$ & .88 \\
\hline Stroke & $29(2.5)$ & $23(2.4)$ & $6(2.9)$ & .72 \\
\hline \multicolumn{5}{|l|}{ Surgical characteristics } \\
\hline Artificial chordae implant & $261(15.2)$ & $211(15.2)$ & $50(15.9)$ & .73 \\
\hline ECC (min) & $60 \pm 30$ & $61 \pm 31$ & $59 \pm 28$ & .44 \\
\hline Interatrial communication & $267(15.6)$ & $222(16.3)$ & $45(14.3)$ & .38 \\
\hline Leaflet plication & $331(19.4)$ & 259 (18.6) & $72(22.9)$ & .08 \\
\hline Maze procedure & $178(10.4)$ & $145(10.4)$ & $33(10.5)$ & .96 \\
\hline Robotic MV repair & $262(15.4)$ & $213(15.3)$ & $49(15.6)$ & .81 \\
\hline Surgical era (2003-2012) & $1073(62.9)$ & $884(63.5)$ & $189(60.2)$ & .26 \\
\hline Triangular resection & $1116(65.5)$ & $902(64.8)$ & $214(68.2)$ & .26 \\
\hline TV repair & $77(4.5)$ & $64(4.6)$ & $13(4.1)$ & .72 \\
\hline Aortic crossclamp time (min) & $43 \pm 22$ & $43 \pm 22$ & $42 \pm 21$ & .43 \\
\hline
\end{tabular}

Data presented as n (\%) or mean \pm standard deviation. $L V E F$, Left ventricular ejection fraction; $B M I$, body mass index; $B S A$, body surface area; COPD, chronic obstructive pulmonary disease; $N Y H A$, New York Heart Association; $E C C$, extracorporeal circulation; $M V$, mitral valve; $T V$, tricuspid valve.

the threshold values of LVESD and right ventricular systolic pressure (RVSP) that offered the greatest discriminatory power in predicting the development of early postoperative LVEF of $<40 \%$. All statistical tests were 2-sided, with the $\alpha$ level set at 0.05 for statistical significance. Analyses were performed using Statistical Analysis Systems, version 9.13, software (SAS institute, Inc, Cary, NC).

\section{RESULTS}

\section{Baseline Clinical Characteristics}

All patients had a preoperative LVEF $>60 \%$ on the preoperative echocardiogram. The baseline characteristics for all patients and those stratified by the presence or absence of postoperative LV dysfunction (EF $<50 \%$ ) are presented in Table 1 . The overall mean age was $59 \pm 13$ years, and 575 of the patients were women $(34 \%)$. Of the 1705 patients with preserved LVEF before surgery, 314 $(18.4 \%)$ developed systolic dysfunction $(\mathrm{LVEF}<50 \%)$ after mitral valve repair. With the exception of a lower prevalence of women $(28 \%$ vs $35 \% ; P=.018)$ and patients with hypertension $(33 \%$ vs $42 \% ; P=.003)$ in those with LV dysfunction, all other baseline and operative variables were comparable between the 2 groups. No differences were found in the surgical techniques used or the ischemic or cardiopulmonary bypass times between those with and without early postoperative LV dysfunction.

To further understand the fate of a subgroup of patients without guideline-based class I or IIA triggers for mitral valve surgery, we identified and analyzed those 585 (34\%) asymptomatic patients with normal preoperative LV function and LVESD $<40 \mathrm{~mm}$, who were also free from atrial fibrillation and pulmonary hypertension (RVSP $<50 \mathrm{~mm} \mathrm{Hg}$ ) at baseline surgery. Of these, 68 patients had evidence of early postmitral valve repair $\mathrm{LV}$ dysfunction $(\mathrm{EF}<50 \%)$ on predismissal echocardiography-representative of $22 \%$ of this "trigger-free" subgroup (68 of 314 with LVEF $<50 \%$ ).

\section{Early Postoperative Clinical Outcomes}

The postoperative clinical course of all patients and those stratified according to postoperative LVEF are outlined in Table 2. Overall, a total of 4 early deaths $(0.2 \%)$ occurred, with no difference in mortality between patients with and without early postoperative LVEF $<50 \%(0.14 \%$ vs $0.64 \% ; P=.10)$. As expected, patients with early LV dysfunction more frequently required postoperative inotropic support $(28.0 \%$ vs $37.3 \%, P=.04)$, although this did not correlate with a prolonged intensive care unit or hospital stay.

\section{Early Changes in Echocardiographic Parameters}

Changes in LVEF, LV dimensions, and pulmonary artery pressure between preoperative and early postoperative assessments were examined and stratified according to the presence or absence of early postoperative LV dysfunction 
TABLE 2. Comparison of postoperative clinical course stratified by early predismissal LVEF

\begin{tabular}{lccc}
\hline & \multicolumn{2}{c}{ EF } & \\
\cline { 2 - 3 } \multicolumn{1}{c}{ Variable } & $\begin{array}{c}\geq \mathbf{5 0} \% \\
(\mathbf{n}=\mathbf{1 3 9 1})\end{array}$ & $\begin{array}{c}<\mathbf{5 0} \% \\
(\mathbf{n}=\mathbf{3 1 4})\end{array}$ & $\begin{array}{c}\boldsymbol{P} \\
\text { value }\end{array}$ \\
\hline Hospital LOS (d) & $5(4-7)$ & $5(4-7)$ & .37 \\
IABP & $10(0.7)$ & $3(0.9)$ & .66 \\
ICU stay (h) & $22.5(18.5-22.5)$ & $23(18.8-26.5)$ & .11 \\
Infection (all) & $47(3.4)$ & $6(1.9)$ & .17 \\
Need for inotropic support & $136(28)$ & $50(37.3)$ & .04 \\
Renal failure & $7(0.5)$ & $4(1.2)$ & .12 \\
Reoperation for bleeding & $32(2.3)$ & $12(3.8)$ & .12 \\
Residual moderate or more MR & $33(2.4)$ & $5(1.5)$ & .35 \\
Stroke & $6(0.4)$ & $1(0.3)$ & .78 \\
\hline
\end{tabular}

Data presented as median (interquartile range) or $\mathrm{n}(\%) . E F$, Ejection fraction; $L O S$, length of stay; $I A B P$, intra-aortic balloon pump; ICU, intensive care unit; $M R$, mitral regurgitation.

(Table 3 and Figure 1). As expected, patients with a postoperative $\mathrm{EF}$ of $<50 \%$ experienced a greater decline in $\mathrm{EF}$ than did those without $\mathrm{LV}$ impairment $(\mathrm{EF} \geq 50 \%$, $-7.6 \%$ vs $\mathrm{EF}<50 \%,-23.5 \% ; P<.001)$. Although changes in LV end-diastolic diameter were comparable between groups $(\mathrm{EF} \geq 50 \%,-6.8 \mathrm{~mm}[-11.8 \%]$ vs $\mathrm{EF}<50 \%,-6.6 \mathrm{~mm}[-10.7 \%] ; P=.54)$, early postoperative LV dysfunction coincided with a significant enlargement in LVESD ( $\mathrm{EF} \geq 50 \%,-0.6 \mathrm{~mm}[-1.7 \%]$ vs $\mathrm{EF}<50 \%,+4.3 \mathrm{~mm}[+11.4 \%] ; P<.001)$ and decrease in pulmonary artery pressure (RVSP, EF $\geq 50 \%,-2.7[-7.1 \%]$ vs $\mathrm{EF}<50 \%,-7.8[-18.1 \%]$; $P<.001)$.

TABLE 3. Comparison of pre- and postoperative echocardiographic data

\begin{tabular}{lccc}
\hline & \multicolumn{2}{c}{$\mathbf{E F}$} & \\
\cline { 2 - 3 } \multicolumn{1}{c}{ Variable } & $\mathbf{5 0} \%(\mathbf{n}=\mathbf{1 3 9 1})$ & $<\mathbf{5 0} \%(\mathbf{n}=\mathbf{3 1 4})$ & $\boldsymbol{P}$ value \\
\hline LVEF (\%) & & & \\
$\quad$ Preoperatively & $66.8 \pm 4.8(66)$ & $65.3 \pm 4.0(65)$ & $<.001$ \\
Postoperatively & $59.2 \pm 5.8(60)$ & $41.8 \pm 6.2(45)$ & $<.001$ \\
Change & $-7.6 \pm 6.8(-8)$ & $-23.5 \pm 7.3(-22)$ & $<.001$ \\
LVEDD (mm) & & & \\
Preoperatively & $57.3 \pm 5.9(57)$ & $61.8 \pm 5.9(62)$ & $<.001$ \\
Postoperatively & $50.3 \pm 5.7(50)$ & $54.6 \pm 6.1(55)$ & $<.001$ \\
Change & $-6.8 \pm 5.1(-7)$ & $-6.6 \pm 4.7(-6)$ & .54 \\
LVESD (mm) & & & \\
Preoperatively & $34.2 \pm 4.6(34)$ & $37.7 \pm 4.4(38)$ & $<.001$ \\
Postoperatively & $33.4 \pm 5.1(33)$ & $41.9 \pm 5.4(42)$ & $<.001$ \\
Change & $0.6 \pm 4.2(-1)$ & $4.3 \pm 4.5(-6)$ & $<.001$ \\
RVSP (mm Hg) & & & \\
Preoperatively & $38 \pm 13.2(34)$ & $43.2 \pm 16.7(39)$ & $<.001$ \\
Postoperatively & $35.3 \pm 9.6(34)$ & $35.5 \pm 9.4(34)$ & .62 \\
Change & $-2.7 \pm 12.6(-1.8)$ & $-7.8 \pm 15.9(-5)$ & $<.001$ \\
\hline Dennnn
\end{tabular}

Data presented as mean \pm standard deviation (median). $L V E F$, Left ventricular ejection fraction; $L V E D D$, left ventricular end-diastolic diameter; $L V E S D$, left ventricular end-systolic diameter; $R V S P$, right ventricular systolic pressure; $E F$, ejection fraction.
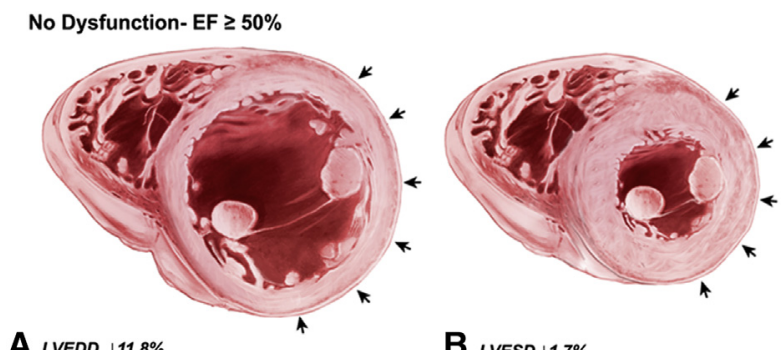

A LVEDD $\downarrow 11.8 \%$

B LVESD $\downarrow 1.7 \%$
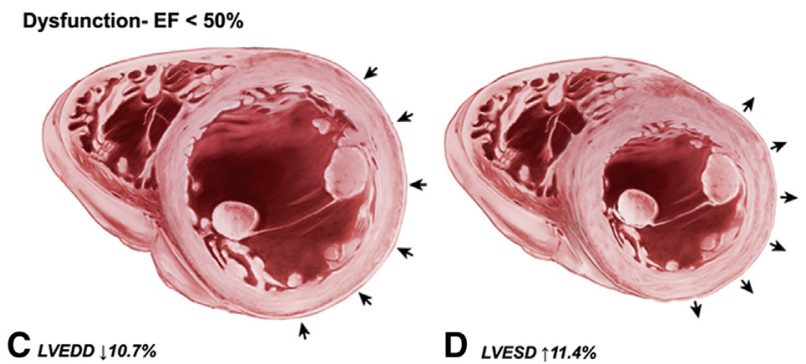

FIGURE 1. Change in left ventricular (LV) end-diastolic diameter $(L V E D D)$ and LV end-systolic diameter (LVESD) between preoperative and early postoperative echocardiograms. Top, Among patients without postoperative LV impairment (ejection fraction $[E F]$ $\geq 50 \%$ ), (A) LVEDD and (B) LVESD declined after mitral valve repair. Bottom, In those with postoperative LV dysfunction (EF $<50 \%$ ), (C) LVEDD decreased and (D) LVESD increased after surgical valve repair.

\section{Long-Term Evolution of LV Size and Function}

Early postoperative LV dysfunction identified immediately after mitral valve repair was found to persist during longitudinal echocardiographic follow-up. Five years after index repair, EF had recovered to preoperative levels of $>60 \%$ in only $31.5 \%$ of the patients with early postoperative $\mathrm{LV}$ dysfunction ( $\mathrm{EF}<50 \%$ ) compared with $65.9 \%$ of those without early LV dysfunction (EF $\geq$ $50 \% ; P<.001 ;$ Figure 2, A). Specifically, EF remained significantly lower in the early dysfunction group, while both the LV end-diastolic diameter and the LVESD were higher up to 10 years following mitral valve repair (Figure 2, B).

\section{Predictors of Early EF $<\mathbf{5 0} \%$}

In an effort to identify factors predisposing patients with preoperative $\mathrm{EF}>60 \%$ to the development of early postoperative LV impairment after mitral valve repair, we further studied the predictors of postoperative LVEF $<50 \%$ (Table 4). After adjustment for influential covariates in multivariable logistic regression analysis, a larger preoperative LVESD (odds ratio [OR], 1.2 per 1 $\mathrm{mm} ; 95 \%$ confidence interval $[\mathrm{CI}], 1.14-1.24 ; P<.001)$ and higher RVSP (OR, 1.03 per $1 \mathrm{~mm} \mathrm{Hg} ; 95 \% \mathrm{CI}$, $1.02-1.04 ; P<.001)$ were independent predictors of postoperative LV dysfunction. 

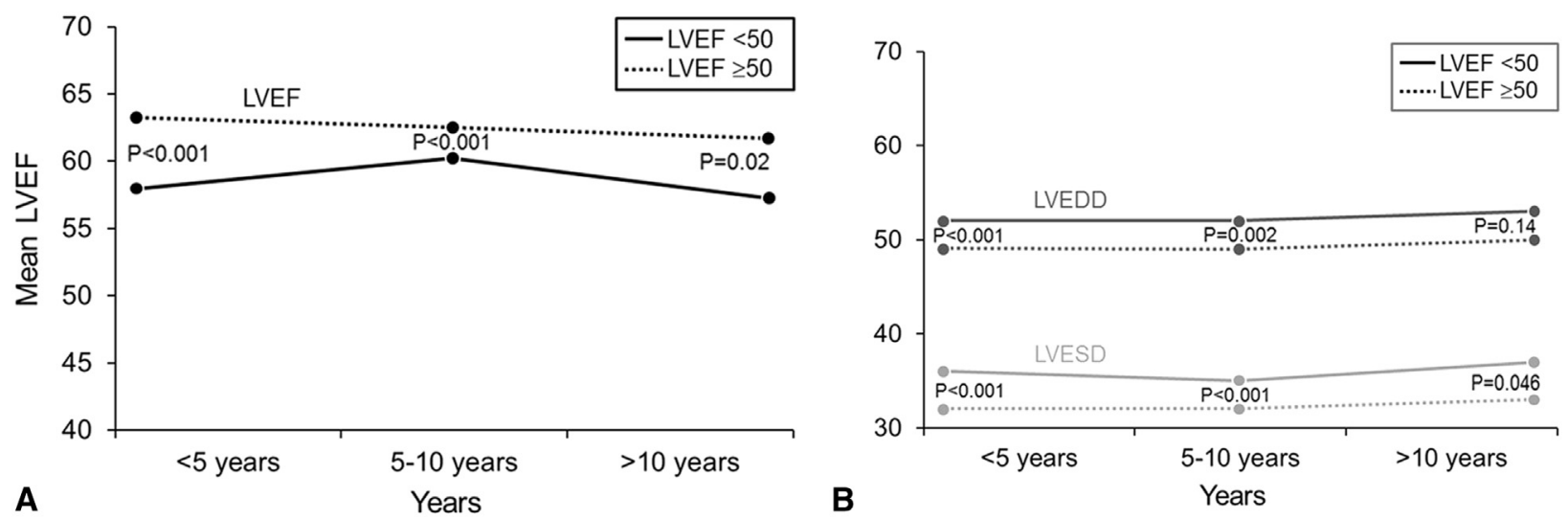

FIGURE 2. Evolution of echocardiographic data during follow-up compared in both groups according to early postoperative left ventricular ejection fraction $(L V E F)<50 \%$ or $\geq 50 \%$ at different time points after mitral valve repair. Comparison of (A) LVEF and (B) left ventricular end-diastolic diameter (LVEDD) (black) and left ventricular end-systolic diameter (LVESD) (gray).

\section{Late Reoperation}

Freedom from reoperation at 5 and 10 years was $96.7 \%$ and $93.8 \%$, respectively. A total of 54 patients $(3.4 \%)$ required a second operation on the mitral valve at a mean of 5 years after the initial repair, corresponding to a linearized rate of reoperation of $0.1 \%$ per year. In 19 patients $(35 \%)$, the valve was re-repaired, and $35(65 \%)$ underwent prosthetic valve replacement. The reasons for reoperation included MR recurrence in 50 patients, systolic anterior motion in 1, and mitral valve endocarditis in 3 . No differences were seen in the reoperation rate between patients with versus without postoperative LV dysfunction $(\mathrm{EF}<50 \% ; P=.37)$.

\section{Long-Term Survival}

The data on long-term survival were $93 \%$ complete. The mean \pm standard deviation follow-up was $5.9 \pm 5.2$ years

TABLE 4. Preoperative predictors of early postoperative LV dysfunction $(\mathbf{E F}<\mathbf{5 0} \%)$

\begin{tabular}{|c|c|c|c|c|}
\hline \multirow{2}{*}{$\begin{array}{c}\text { Preoperative } \\
\text { data }\end{array}$} & $\begin{array}{c}\text { Univariate } \\
\text { analysis }\end{array}$ & \multirow[b]{2}{*}{$P$ value } & $\begin{array}{c}\text { Multivariate } \\
\text { model }\end{array}$ & \multirow{2}{*}{$\begin{array}{c}P \\
\text { value }\end{array}$} \\
\hline & OR $(95 \%$ CI $)$ & & OR $(95 \%$ CI $)$ & \\
\hline Female sex & $0.72(0.55-0.95)$ & .018 & & \\
\hline Hypertension & $0.68(0.52-0.87)$ & .002 & & \\
\hline Greater LVEF & $0.93(0.9-0.95)$ & $<.001$ & & \\
\hline Greater LVEDD & $1.14(1.11-1.16)$ & $<.001$ & & \\
\hline Greater LVESD & $1.18(1.14-1.23)$ & $<.001$ & $1.2(1.14-1.24)$ & $<.001$ \\
\hline Greater LAD & $1.05(1.02-1.08)$ & $<.001$ & & \\
\hline Greater LV mass & $1.01(1-1.01)$ & $<.001$ & & \\
\hline $\begin{array}{l}\text { Greater ERO } \\
\text { (PISA) }\end{array}$ & $3.52(2.15-5.76)$ & $<.001$ & & \\
\hline Greater RVSP & $1.02(1.01-1.03)$ & $<.001$ & $1.03(1.02-1.04)$ & $<.001$ \\
\hline
\end{tabular}

$L V$, Left ventricular; $O R$, odds ratio; $C I$, confidence interval; $L V E F$, left ventricular ejection fraction; $L V E D D$, left ventricular end-diastolic diameter; $L V E S D$; left ventricular end-systolic diameter; $L A D$, left atrial diameter; $E R O$, effective regurgitant orifice; PISA, proximal isovelocity surface area; $R V S P$, right ventricular systolic pressure. (maximum, 20.2). A total of 170 patients $(9.9 \%)$ died during the follow-up period at a mean age of 78 years. Overall survival for all patients was $95 \%, 85 \%$, and $70.8 \%$ at 5,10 , and 15 years, respectively. When considering late outcomes in patients with early postoperative $\mathrm{EF} \geq 50 \%$ versus $<50 \%$, survival was comparable between the groups at all measurement points ( 5 years, $96 \%$ vs $95 \%$; 10 years, $89 \%$ vs $86 \% ; 15$ years, $79 \%$ vs $73 \% ; P=.65$ ).

To further evaluate the relationship between the differing severities of early postoperative LV dysfunction and late mortality, we generated spline functions, studying the predismissal EF as a continuous variable (Figure 3). We found that progressively poorer early postoperative $\mathrm{EF}$ correlated with an increased hazard of late death. In particular, late survival was significantly less in patients with a postoperative $\mathrm{EF}<40 \%(\mathrm{n}=71)$ at dismissal from hospital compared with those with an LVEF of $\geq 40 \%(\mathrm{n}=1634 ; 5$ year, $96 \%$ vs $93 \% ; 10$ years, $87 \%$ vs $75 \% ; 15$ years, $75 \%$ vs $61 \% ; P=.008$; Figure 4 ).

Understanding that more severe degrees of early postoperative LV dysfunction were influential, we further studied the univariate and multivariate predictors of late all-cause mortality. After controlling for the confounding effects of older age (hazard ratio [HR], 1.1; 95\% CI, 1.07-1.1; $P<.001$ ), hypertension (HR, 1.4; 95\% CI, 1.0-1.8; $P=.036)$, New York Heart Association class III or IV (HR, $1.7 ; 95 \% \mathrm{CI}, 1.2-2.3 ; P<.001$ ), and preoperative atrial fibrillation (HR, 2.3; 95\% CI, 1.3-4.2; $P=.005$ ), an early postoperative LVEF of $<40 \%$ was associated with a $70 \%$ increase in the risk of late postoperative mortality (HR, $1.7 ; 95 \%$ CI, 1.03-2.92; $P=.037$; Table 5).

\section{Predictors of Early Postoperative EF $<\mathbf{4 0} \%$}

Having identified an $\mathrm{EF}$ of $<40 \%$ as a potentially important threshold for an increased hazard of late death, we modeled the preoperative determinants of developing this degree of postoperative LV dysfunction immediately 
TABLE 5. Univariate and multivariate predictors of all-cause long-term mortality

\begin{tabular}{|c|c|c|c|c|}
\hline & Univariate analysis & & Multivariate model & \\
\hline Preoperative data & OR $(95 \%$ CI $)$ & $P$ value & OR $(95 \% \mathrm{CI})$ & $P$ value \\
\hline Age & $1.10(1.08-1.11)$ & $<.001$ & $1.09(1.07-1.11)$ & $<.001$ \\
\hline Female sex & $1.32(0.97-1.79)$ & .074 & & \\
\hline Diabetes & $1.55(0.64-3.77)$ & .337 & & \\
\hline Hypertension & $1.78(1.32-2.40)$ & $<.001$ & $1.38(1.02-1.87)$ & .036 \\
\hline NYHA class III-IV & $2.58(1.89-3.53)$ & $<.001$ & $1.71(1.25-2.35)$ & .001 \\
\hline Preoperative atrial fibrillation & $4.88(2.75-8.65)$ & $<.001$ & $2.33(1.39-4.19)$ & .005 \\
\hline $\mathrm{ECC}(\mathrm{min})$ & $1.00(1.00-1.01)$ & .078 & & \\
\hline Aortic crossclamp time (min) & $1.00(1.00-1.01)$ & .473 & & \\
\hline TV repair & $3.75(2.26-6.20)$ & $<.001$ & & \\
\hline Maze procedure & $1.75(1.11-2.77)$ & .017 & & \\
\hline Preoperative RVSP (mm Hg) & $1.03(1.02-1.04)$ & $<.001$ & & \\
\hline $\mathrm{LAD}(\mathrm{mm})$ & $1.03(1.01-1.05)$ & .006 & & \\
\hline Preoperative LVESD & $0.99(0.93-1.06)$ & .824 & & \\
\hline Preoperative LVEDD & $0.99(0.95-1.04)$ & .780 & & \\
\hline Preoperative MR ERO & $0.44(0.20-0.96)$ & .038 & & \\
\hline Preoperative LV mass & $1.00(1.00-1.00)$ & .337 & & \\
\hline Preoperative RVSP $\geq 38 \mathrm{~mm} \mathrm{Hg}$ & $2.94(1.93-4.48)$ & $<.001$ & & \\
\hline Postoperative LVEF < $40 \%$ & $1.99(1.19-3.33)$ & .009 & $1.74(1.03-2.92)$ & .037 \\
\hline
\end{tabular}

$O R$, Odds ratio; $C I$, confidence interval; $N Y H A$, New York Heart Association; $E C C$, extracorporeal circulation; $T V$, tricuspid valve; $R V S P$, right ventricular systolic pressure; $L A D$, left atrial diameter; $L V E S D$, left ventricular end-systolic diameter; $L V E D D$, left ventricular end-diastolic diameter; $M R E R O$, mitral regurgitation effective regurgitant orifice; $L V E F$, left ventricular ejection fraction; $L V$, left ventricular.

after mitral valve repair. Using multivariable logistic regression analyses, we were able to establish that a larger preoperative LVESD and greater preoperative RVSP were both influential $(P<.001$ for both). Through multiple logistic regression analyses, we identified thresholds of LVESD $>36 \mathrm{~mm}$ and RVSP $>49$ as having the greatest discriminatory power in predicting a postoperative EF of $<40 \%$ (Table 6).

\section{DISCUSSION}

We studied early postoperative LV impairment in a homogeneous population of patients with severe degenerative

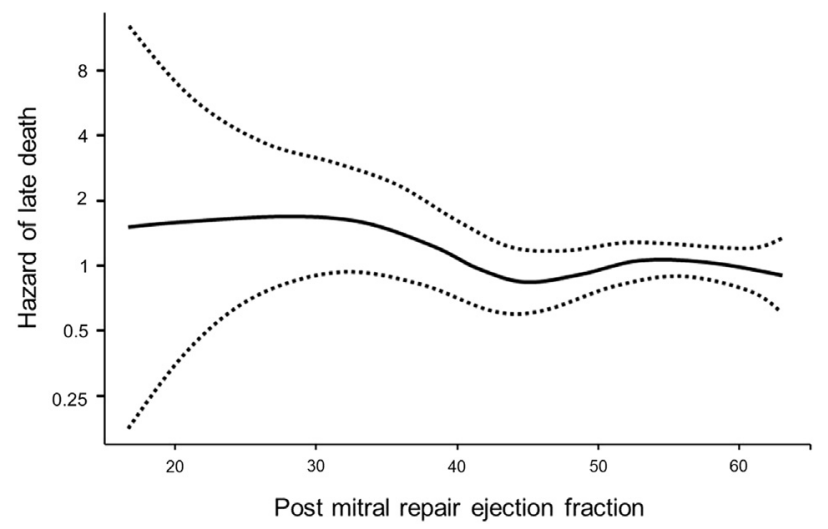

FIGURE 3. Relationship between early ejection fraction (EF) after mitral valve repair and hazard of late death. Hazard ratio and $95 \%$ confidence intervals (dotted lines) for the risk of overall mortality, with the left ventricular EF represented as a spline function. Lower postoperative EF was associated with a greater late risk of late death.
MR and preserved LV function (EF $>60 \%$ ) undergoing mitral valve repair. We found that approximately 1 in 5 patients developed early LV dysfunction ( $\mathrm{EF}<50 \%$ ). Of these patients, one fifth were free of typical class I or IIA indications prior to surgery. Importantly and contrary to the current perception, only 1 in 3 patients with postoperative LV impairment experience recovery of EF to preoperative levels at 5 years after valve repair. Although an EF threshold of $<50 \%$ on predismissal echocardiogram did not appear to affect late survival in the present analysis, leaving the hospital with an $\mathrm{EF}$ of $<40 \%$ did independently predict a $70 \%$ increase in the hazard of late death. Moreover, we have demonstrated that a preoperative RVSP $>49 \mathrm{~mm} \mathrm{Hg}$ and LVESD

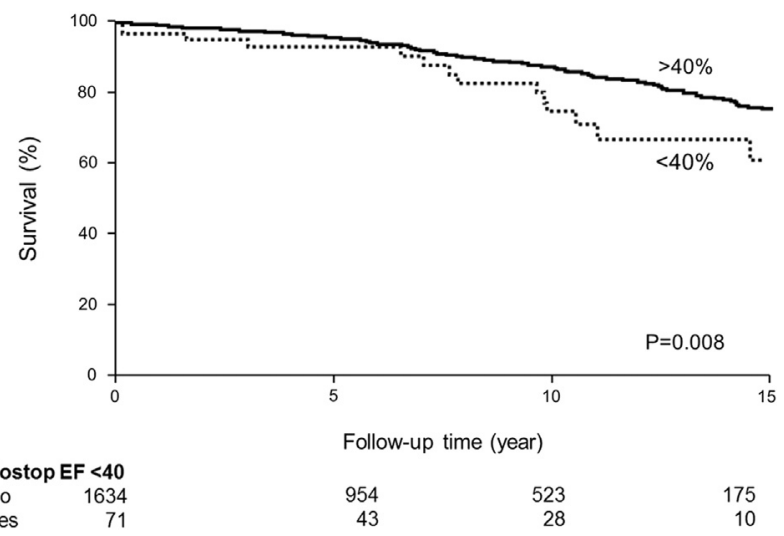

FIGURE 4. Comparison of overall long-term survival stratified by early left ventricular dysfunction (threshold left ventricular ejection fraction $[E F]<40 \%)$. 
TABLE 6. Preoperative predictors of early postoperative LVEF $<40 \%$

\begin{tabular}{llcc}
\hline & Univariate analysis & & Multivariate model \\
\cline { 2 - 3 } Preoperative data & OR $(\mathbf{9 5} \% \mathbf{C I})$ & $\boldsymbol{P}$ value & \multicolumn{1}{c}{ OR $(\mathbf{9 5} \% \mathbf{C I})$} \\
\hline Greater LV mass & $1.01(1.01-1.01)$ & $<.001$ & \\
Greater LAD & $1.07(1.03-1.11)$ & $<.001$ & \\
Greater LVEDD & $1.19(1.14-1.25)$ & $<.001$ & \\
NYHA class III or IV & $1.94(1.2-3.14)$ & .007 & \\
Atrial fibrillation & $2.66(1.32-5.35)$ & $<.006$ & $4.40(2.35-8.23)$ \\
RVSP $>$ 49 mm Hg & $4.57(2.54-8.23)$ & $<.001$ & \\
Higher ERO (PISA) & $5.00(2.4-10.4)$ & $<.001$ & $6.46(3.31-13.61)$ \\
LVESD $>36$ mm Hg & $5.93(3.15-11.97)$ & $<.001$ \\
\hline
\end{tabular}

$O R$, Odds ratio; $C I$, confidence interval; $L V$, left ventricular; $L A D$, left atrial diameter; $L V E D D$, left ventricular end-diastolic diameter; $N Y H A$, New York Heart Association; $R V S P$, right ventricular systolic pressure; ERO, effective regurgitant orifice; PISA, proximal isovelocity surface area; $L V E S D$, left ventricular end systolic diameter.

$>36 \mathrm{~mm}$ are associated with a 4.4- and 6.5-fold increased risk of developing early postoperative $\mathrm{LVEF}<40 \%$, respectively. Collectively, these data suggest that caution must be exercised during "watchful waiting" for patients with severe MR based on the false reassurance of "preserved" preoperative LVEF alone.

As has been demonstrated in previous studies, ${ }^{10}$ we observed a decline in LVEF after surgical correction of MR. It was somewhat surprising, however, that this decrease was also identified in a heretofore "understudied" population with "preserved" preoperative myocardial function. Although an early postoperative decrease in EF between $5 \%$ to $10 \%$ in the nonimpaired ventricle after mitral repair can be explained as a volumetric adjustment to the elimination of the proportion of total ventricular ejection contributing to regurgitation, ${ }^{5,8}$ greater postoperative decreases in EF have been associated with the markers of degenerative MR chronicity, including myocardial fibrosis, hypertrophy, and adverse remodeling. ${ }^{8,18-20}$ Although tempting to impugn surgical intervention as the cause of the deterioration, it is noteworthy that the surgical techniques and operative durations were indistinguishable between the groups with and without early dysfunction, indicating that the myocardial predisposition for LV deterioration was present before the intervention. Importantly, in our present series, patients in both groups had a clinically similar (although statistically different) preoperative EF $(66.8 \%$ vs $65.3 \%$, $P<.001$ ), cautioning against reliance on this nonspecific trigger in determining the extent of myocardial impairment and timing of surgical intervention. The degree of preoperative left-sided heart chamber enlargement appeared influential, as previously reported. ${ }^{8}$ Pulmonary hypertension in patients with MR even in the setting of a "preserved" EF is a frequent finding ${ }^{21}$ that can persist even after repair. ${ }^{22}$ In agreement with Varghese and colleagues, ${ }^{10}$ we found that greater degrees of pulmonary hypertension were associated with poorer early postoperative EF. Our study differed, however, in that we focused on a population of patients without ischemic burden and with a preoperative $\mathrm{EF}>60 \%$.
In our present report, LV function returned to normal levels $(>60 \%)$ in only one third of the patients with an early postoperative $\mathrm{EF}<50 \%$ compared with two thirds of those without ventricular impairment after surgery; thereby, dispelling the commonly held notion that EF "always" recovers after successful elimination of MR. Despite chordal preservation and short crossclamp times, markers of increased MR chronicity preoperatively were associated with impaired long-term capacity for reverse LV remodeling. Eccentric myocardial hypertrophy, fibrosis, ${ }^{23}$ and inflammatory cascades could all potentially be associated with this phenomenon and can only be prevented by mechanical elimination of MR after successful mitral valve repair.

The historic work of Crawford and colleagues ${ }^{11}$ identified an $\mathrm{EF}<50 \%$ after surgical correction of MR as deleterious to long-term survival. That series included mixed etiologies of mitral valve disease (eg, ischemic, rheumatic) and those undergoing valve replacement. We have identified, for the first time to our knowledge, that more severe postrepair LV dysfunction $(\mathrm{EF}<40 \%)$ could be a potent adverse prognostic marker in patients with isolated degenerative MR and, further, that an LVESD $>36 \mathrm{~mm}^{24}$ and an RVSP > $49 \mathrm{~mm} \mathrm{Hg}$ are important predictors of encountering this degree of impairment after degenerative mitral valve repair. Even after controlling for potential confounders, an $\mathrm{EF}<40 \%$ at dismissal from hospital after mitral valve repair independently predicted a $70 \%$ increase in late mortality risk. This important finding cautions against the reassurance of patients that watchful waiting is safe in the presence of preserved ventricular function and severe degenerative MR.

An increasing body of clinical comparative effectiveness data supports the favorable long-term survival and diminished heart failure risk associated with earlier surgical intervention in patients with severe degenerative MR. 2,20,28,29 The argument for the earlier referral of asymptomatic patients with severe degenerative MR in the absence of classic guideline-based triggers hinges, however, on the provision of a valve repair likelihood of 
$>95 \%$ to $99 \%$ for all prolapse categories, ${ }^{16,30}$ with a mortality and/or morbidity risk of $<1 \%$. The clinical relevance of the present findings include highlighting the need for (1) accurate echocardiographic quantification of severe MR in all patients with a systolic murmur, (2) participation in a data-driven discussion regarding the timing of surgery in those with severe MR and an $\mathrm{EF}>60 \%$ (drawing particular attention to recent data addressing the risk/benefit ratio of early mitral valve repair vs watchful waiting); and (3) advising caution in delaying mitral valve repair in the presence of an LVESD of 35 to $40 \mathrm{~mm}$ or RVSP of 40 to $50 \mathrm{~mm} \mathrm{Hg}$. Falsely reassuring patients with severe MR that watchful waiting is safe on the basis of a normal EF alone unnecessarily exposes them to the risk of LV dysfunction and a survival disadvantage when EF after MR correction is $<40 \%$.

Echocardiographically assessed LVEF is ubiquitous in clinical practice and is deeply embedded in contemporary guideline-based consensus statements ${ }^{31}$; however, the use of it alone as a single modality for LV function assessment is falsely reassuring and problematic. Which other markers might be useful in informing the optimal timing of surgical intervention? Although our mitral valve repair practice has evolved to the point that most patients currently undergoing isolated mitral valve repair for severe degenerative MR are asymptomatic and without triggers, patients with MR who were detected late in their disease course or sent from outside institutions where watchful waiting was considered to be "safe" were also included in the present series. Thus, even in the 585 patients $(34 \%$ of our entire population) who were free of both class I (New York Heart Association class III or IV symptoms, EF $\leq 60 \%$, or LVESD > $>40 \mathrm{~mm}$ ) and class II (atrial fibrillation or pulmonary hypertension) triggers for intervention, $12 \%$ (68 of 585) still developed early postoperative LV dysfunction ( $\mathrm{LVEF}<50 \%$ ). This finding emphasizes the point that even in this "favorable" population who underwent "very-early" mitral valve repair, chronic severe MR could already have resulted in irreversible myocardial damage and deleterious clinical consequences. The capacity for nascent modalities such as myocardial strain, ${ }^{25}$ hormonal activation, ${ }^{26}$ and exercise capacity ${ }^{27}$ to better identify those at increased risk of LV dysfunction and/or diminished late survival is presently under investigation at several centers worldwide. In addition to the long-term survival implications, the clinical importance of identifying those with limited potential for myocardial recovery lies in facilitating both the initiation of aggressive medical therapy and also increased frequency of clinical and echocardiographic surveillance.

\section{Study Limitations}

The present study was subject to the limitations inherent in a nonrandomized observational series. The patients in the present study were necessarily selected because of their willingness and availability to undergo clinical and echocardiographic surveillance. We were unable to obtain follow-up echocardiograms for each and every patient in our population encompassing a surgical experience spanning $>2$ decades. Additionally, owing to the particular referral pattern in our practice, accurate tracking of death in patients from foreign countries was limited by laws regulating the release of death certificates. Despite widespread availability of echocardiography as a guideline-sanctioned measure of LV function, the technology is limited in its ability to detect latent LV dysfunction. Future studies using exercise-induced myocardial strain assessment and magnetic resonance imaging will be informative.

\section{CONCLUSIONS}

Although mitral valve repair for degenerative disease can be performed with very low mortality in patients with "normal" preoperative EF, early postoperative LV dysfunction is not uncommon. Importantly, early myocardial impairment might be irreversible and associated with increased long-term mortality risk. A preserved preoperative $\mathrm{EF}>60 \%$ should, therefore, not provide false reassurance of the maintained capacity for restoration of LV function or normalization of survival when mitral valve repair is delayed. These data indicate that surgical intervention before the onset of excessive LV dilation, pulmonary hypertension, or symptoms appears warranted.

The authors wish to thank Judy Lenoch for assistance in data retrieval and David Hodge for the statistical assessment.

\section{References}

1. Kang DH, Park SJ, Sun BJ, Cho EJ, Kim DH, Yun SC, et al. Early surgery versus conventional treatment for asymptomatic severe mitral regurgitation: a propensity analysis. J Am Coll Cardiol. 2014;63:2398-407.

2. Suri RM, Vanoverschelde JL, Grigioni F, Schaff HV, Tribouilloy C, Avierinos JF, et al. Association between early surgical intervention vs watchful waiting and outcomes for mitral regurgitation due to flail mitral valve leaflets. JAMA 2013;310:609-16.

3. Otto CM. Surgery for mitral regurgitation: sooner or later? JAMA. 2013;310 587-8.

4. Nishimura RA, Otto CM, Bonow RO, Carabello BA, Erwin JP III, Guyton RA, et al. 2014 AHA/ACC guideline for the management of patients with valvular heart disease: executive summary: a report of the American College of Cardiology/American Heart Association Task Force on Practice Guidelines. Circulation. 2014;129:2440-92

5. Witkowski TG, Thomas JD, Delgado V, van Rijnsoever E, Ng AC, Hoke U, et al. Changes in left ventricular function after mitral valve repair for severe organic mitral regurgitation. Ann Thorac Surg. 2012;93:754-60.

6. Tribouilloy C, Grigioni F, Avierinos JF, Barbieri A, Rusinaru D, Szymanski C, et al. Survival implication of left ventricular end-systolic diameter in mitral regurgitation due to flail leaflets a long-term follow-up multicenter study. J Am Coll Cardiol. 2009;54:1961-8.

7. Topilsky Y, Suri R, Schaff HV, Enriquez-Sarano M. When to intervene for asymptomatic mitral valve regurgitation. Semin Thorac Cardiovasc Surg. 2010;22:216-24

8. Suri RM, Schaff HV, Dearani JA, Sundt TM III, Daly RC, Mullany CJ, et al. Determinants of early decline in ejection fraction after surgical correction of mitral regurgitation. J Thorac Cardiovasc Surg. 2008;136:442-7. 
9. Enriquez-Sarano M, Tajik AJ, Schaff HV, Orszulak TA, McGoon MD, Bailey KR, et al. Echocardiographic prediction of left ventricular function after correction of mitral regurgitation: results and clinical implications. J Am Coll Cardiol. 1994;24:1536-43.

10. Varghese R, Itagaki S, Anyanwu AC, Milla F, Adams DH. Predicting early left ventricular dysfunction after mitral valve reconstruction: the effect of atrial fibrillation and pulmonary hypertension. J Thorac Cardiovasc Surg. 2014;148: 422-7.

11. Crawford M, Souchek J, Oprian CA, Miller DC, Rahimtoola S, Giacomini JC, et al. Determinants of survival and left ventricular performance after mitral valve replacement. Circulation. 1990;81:1173-81.

12. Marciniak A, Sutherland GR, Marciniak M, Kourliouros A, Bijnens B, Jahangiri M. Prediction of postoperative left ventricular systolic function in patients with chronic mitral regurgitation undergoing valve surgery-the role of deformation imaging. Eur J Cardiothorac Surg. 2011;40:1131-7.

13. Agricola E, Oppizzi M, De Bonis M, Maisano F, Toracca L, Bove T, et al. Multiplane transesophageal echocardiography performed according to the guidelines of the American Society of Echocardiography in patients with mitral valve prolapse, flail, and endocarditis: diagnostic accuracy in the identification of mitral regurgitant defects by correlation with surgical findings. $J$ Am Soc Echocardiogr. 2003;16:61-6.

14. Brown ML, Schaff HV, Li Z, Suri RM, Daly RC, Orszulak TA. Results of mitral valve annuloplasty with a standard-sized posterior band: is measuring important? J Thorac Cardiovasc Surg. 2009;138:886-91.

15. Suri R, Orszulak TA. Triangular resection for repair of mitral regurgitation due to degenerative disease. Oper Techn Thorac Cardiovasc Surg. 2005;8: 194-9.

16. Suri RM, Burkhart HM, Daly RC, Dearani JA, Park SJ, Sundt TM III, et al. Robotic mitral valve repair for all prolapse subsets using techniques identical to open valvuloplasty: establishing the benchmark against which percutaneous interventions should be judged. J Thorac Cardiovasc Surg. 2011;142:970-9.

17. Eilers PHC, Marx BD. Flexible smoothing with B-splines and penalties. Stat Sci. 1996;11:89-121.

18. Tribouilloy C, Rusinaru D, Szymanski C, Mezghani S, Fournier A, Lévy F, et al. Predicting left ventricular dysfunction after valve repair for mitral regurgitation due to leaflet prolapse: additive value of left ventricular end-systolic dimension to ejection fraction. Eur J Echocardiogr. 2011;12:702-10.

19. Suri RM, Schaff HV, Dearani JA, Sundt TM, Daly RC, Mullany CJ, et al. Recovery of left ventricular function after surgical correction of mitral regurgitation caused by leaflet prolapse. J Thorac Cardiovasc Surg. 2009;137:1071-6.

20. Stulak JM, Suri RM, Dearani JA, Burkhart JM, Sundt JM III, Enriquez-Sarano M, et al. Does early surgical intervention improve left ventricular mass regression after mitral valve repair for leaflet prolapse? J Thorac Cardiovasc Surg. 2011; $141: 122-9$.

21. Alexopoulos D, Lazzam C, Borrico S, Fielder L, Ambrose JA. Isolated chronic mitral regurgitation with preserved systolic left ventricular function and severe pulmonary hypertension. J Am Coll Cardiol. 1989;14:319-22.

22. Goldstone AB, Chikwe J, Pinney SP, Anyanwu AC, Funt SA, Polanco A, et al. Incidence, epidemiology, and prognosis of residual pulmonary hypertension after mitral valve repair for degenerative mitral regurgitation. Am J Cardiol. 2011;107: 755-60.

23. Conway MA, Bottomley PA, Ouwerkerk R, Radda GK, Rajagopalan B. Mitral regurgitation: impaired systolic function, eccentric hypertrophy, and increased severity are linked to lower phosphocreatine/ATP ratios in humans. Circulation. 1998;97:1716-23.

24. Suri RM, Schaffe HV, Dearani JA, Sundt TM III, Daly RC, Mullany CJ, et al Survival advantage and improved durability of mitral repair for leaflet prolapse subsets in the current era. Ann Thorac Surg. 2006;82:819-27.

25. Witkowski TG, Thomas JD, Debonnaire PJ, Delgado V, Hoke U, Ewe SH, et al. Global longitudinal strain predicts left ventricular dysfunction after mitral valve repair. Eur Heart J Cardiovasc Imaging. 2013;14:69-76.

26. Klaar U, Gabriel H, Bergler-Klein J, Pernicka E, Heger M, Mascherbauer J, et al. Prognostic value of serial B-type natriuretic peptide measurement in asymptomatic organic mitral regurgitation. Eur J Heart Fail. 2011;13:163-9.

27. Messika-Zeitoun D, Johnson BD, Nkomo V, Avierinos JF, Allison TG, Scott C, et al. Cardiopulmonary exercise testing determination of functional capacity in mitral regurgitation: physiologic and outcome implications. J Am Coll Cardiol. 2006;47:2521-7.

28. Kang DH, Kim JH, Rim JH, Kim MJ, Yun SC, Song JM, et al. Comparison of early surgery versus conventional treatment in asymptomatic severe mitral regurgitation. Circulation. 2009;119:797-804.
29. Chenot F, Montant P, Vancraeynest D, Pasquet A, Gerber B, Noirhomme PH, et al. Long-term clinical outcome of mitral valve repair in asymptomatic severe mitral regurgitation. Eur J Cardiothorac Surg. 2009;36:539-45.

30. Castillo JG, Anyanwu AC, Fuster V, Adams DH. A near 100\% repair rate for mitral valve prolapse is achievable in a reference center: implications for future guidelines. J Thorac Cardiovasc Surg. 2012;144:308-12.

31. Nishimura RA, Otto C. 2014 ACC/AHA valve guidelines: earlier intervention for chronic mitral regurgitation. Heart. 2014;100:905-7.

\section{Discussion}

Dr David H. Adams (New York, NY). Rakesh, I really enjoyed this presentation and your leadership in the field. So much of the evidence base that serves as a foundation for the American College of Cardiology/American Heart Association (ACC/AHA) guidelines have originated from the Mayo experience, and your new data are provocative and will have implications for future guidelines. Let us explore a few details.

My first question is, what led you to focus on patients with an $\mathrm{EF}>60 \%$ instead of all-comers, given that the surgical trigger point around $60 \%$ has been an established criterion in the ACC/AHA guidelines for the past several years based on historical data from the Mayo? I imagine there was some curiosity among your colleagues about this study in the first place, because these results could well redefine what we have held true for the past few decades.

Dr Suri. Thank you, Dr Adams, and we would like to acknowledge your international leadership and contributions to this field.

You make an important point, and that is, why should we consider operating in advance of established guidelines?

Comparative effectiveness research recently published, including the Mitral Regurgitation International DAtabase (MIDA) analysis late last year and the study by Kang and colleagues several weeks ago, both point us in the direction of understanding to a greater degree the ability of early mitral valve repair-which is low risk and associated with low morbidity in the modern era-to improve late outcomes. This is true, in terms of both enhanced late survival and freedom from heart failure many years after early mitral valve repair. A growing population of patients thus present to us at Mayo Clinic without the typical class I or IIA triggers for operation and elect to undergo early correction of severe degenerative MR, cognizant of these benefits. Most, if not all, of these patients, have a "normal" preoperative EF.

But what struck us in this particular population of patients was that, despite having what was thought to be "normal" ventricular function before surgery, a proportion of these patients develop profound LV dysfunction immediately after separation from cardiopulmonary bypass. Typically, these individuals require inotropic support for a couple of days and leave the hospital with an $\mathrm{EF}$ of $<50 \%$. The first question that many have asked is, "What happened during the operation?"

Well, we went back to our operative notes and found no important technical differences in the course of the operation. These are often patients who have 20- or 30-minute crossclamp times and successful mitral valve repair operations with none-totrivial residual MR, yet they developed LV dysfunction. This led us to ask "why" and "when" and to further investigate this 
heretofore understudied population of patients.

Dr Adams. It is a point Carpentier made, and it is so relevant to all of us. Often, our patient experience triggers our trying to answer a question.

My next point is about the current guidelines for the end-systolic diameter of $40 \mathrm{~mm}$ and the estimated pulmonary artery pressure of $\geq 55 \mathrm{~mm} \mathrm{Hg}$ as the cutoff for decision-making for surgical intervention. Why did you choose the trigger levels you highlighted today? How did you settle on the end-systolic diameter of $36 \mathrm{~mm}$, for example, as a potential trigger instead of another number?

Dr Suri. Thank you. That is a very good question. As those in the room know who have worked to identify cutpoints, it is a very statistically "intensive" process. I can summarize by saying we worked closely with our statisticians to construct multiple logistic regression analyses and identified threshold values of LVESD and RVSP that predicted the development of postoperative LVEF $<40 \%$ with the greatest discriminatory power. We assessed this using the areas under the receiver operating characteristic curves.

The next point to emphasize is that these are not magical numbers or cutpoints. We do not imagine they will necessarily be embedded into future iterations of the recommendations guiding treatment of all patients with degenerative MR. However, we can state that these thresholds appear influential in predicting postoperative LV dysfunction specifically in the present population we studied with an EF $>60 \%$ on the preoperative echocardiogram. These results will hopefully prompt initiation of the next generation of clinical trials to further study advanced indications for the performance of mitral valve repair, specifically in patients with echocardiographically documented severe MR by proximal isovelocity surface area measurements.

Dr Adams. Let us continue on that theme and talk about additive value now. Can you tell us whether you examined the outcomes of patients who had the combination of an elevated end-systolic diameter of $36 \mathrm{~mm}$ and pulmonary pressure of $\geq 45$ $\mathrm{mm} \mathrm{Hg}$ ? A few years ago, Triboully and colleagues emphasized the additive value of near trigger points, not exceeding one but just near trigger points, and I wonder if you have done this exploratory analysis?

Dr Suri. That is a very good question, and I would say the results of the present study have piqued our interest in pursuing additional subset analyses. Just "back-of-napkin" calculations, we have combined those 2 trigger points-LVESD $>36 \mathrm{~mm}$ and RVSP $>49 \mathrm{~mm} \mathrm{Hg}$ - and arrived at an odds ratio that was greater in conjunction than for each of them separately. We estimated an approximately eightfold greater risk of developing postoperative $\mathrm{LVEF}<40 \%$ when both of those trigger points were observed prior to mitral valve repair.

Dr Adams. This is really important, because we need to move away from single-number triggers and start thinking about the combination and your trigger points, and I expect when you do that analysis, we might be even lower than 36 or 45 in combination.

My next point is about your bypass times, which you mentioned were extremely short. I find that actually very sobering, in particular, as the guidelines are moving us toward asymptomatic intervention in class IIA indications. Do you have any thoughts about that in terms of why this is happening or what we need to learn for the future?
Dr Suri. That is a great question. As I mentioned previously, once a patient with asymptomatic MR leaves the operating room with no MR but with an EF that is $\leq 40 \%$, the first question that people ask the surgeon is, "What happened, how long was the crossclamp time?" Well, what struck us was that the crossclamp and bypass times were not different between those with and without postoperative LV dysfunction. In other words, the predisposition for $\mathrm{LV}$ dysfunction was set before the performance of mitral valve repair, and that is something we all need to recognize and investigate further.

Dr Adams. My last comment is about the clinical implications of your results for our daily practice. Your thoughts about strain, other analyses of the ventricle outside of the ejection fraction? Finally, how are you currently treating patients with a lower EF after mitral valve repair for degenerative disease?

Thank you again for this provocative analysis.

Dr Suri. Thank you, Dr Adams. To be very quick with the response for the sake of time, the next wave of prognostic markers that will be investigated to help advance our understanding of the pathophysiologic consequences of severe chronic degenerative MR will include assessment of brain natriuretic peptide, exercise testing, LV strain, and left atrial chamber size. We, and others around the world, are currently investigating the sensitivity and specificity of these modalities in attempting to elucidate the ideal timing of mitral valve repair in asymptomatic patients in advance of the onset of guideline-based triggers.

What do we do when we document LV dysfunction on the predismissal echocardiogram? Dr Schaff, myself, and my colleagues at Mayo Clinic, when we are faced with a patient who has an $\mathrm{EF}<50 \%$ after mitral valve repair, we generally institute angiotensin converting enzyme inhibitor therapy at dismissal. We also consider those patients candidates for increased intensity of medical and echocardiographic surveillance when they return to their home community.

Upon discovery of profound postoperative dysfunction, an EF of $<40 \%$, after mitral valve repair in a patient with a previously normal EF, we consider involving our heart failure colleagues before dismissal from the hospital, because we believe these patients should be monitored with even greater frequency.

Dr Niv Ad (Falls Church, Va). Rakesh, I enjoyed your presentation. I think, on the same line, it is important to understand what your definition of a successful repair is, because we all know that leaving the operating room without MR is not always enough. Also, what happened to the patient who died earlier or had a greater mortality rate with regard to their mitral repair status? Was the successful repair stable throughout the study or did you have grade $\geq 2 \mathrm{MR}$ in this group that died?

Dr Suri. There was $0.2 \%$ mortality in this population, so 4 deaths in the whole study. It was very low.

To the point about what constitutes a successful repair, at Mayo Clinic, we rely on our echocardiographic assessment, separating from cardiopulmonary bypass with trivial-to-mild or less MR, and a gradient that is $<3$, typically. Is that what you mean?

Dr Ad. We all know that leaving the operating without MR is not necessarily enough, and it is not definitely reflective of the long-term success of the mitral valve repair.

Dr Suri. So echocardiographic predictors?

Dr Ad. Yes. 
Dr Suri. Yes, we scrutinize a few other important features on the echocardiogram, such as coaptation depth, and confirm smooth nonturbulent outflow, the absence of systolic anterior motion, and the absence of regional wall motion abnormalities.

Those are the typical things. Is that what you are getting at?

Dr del Nido. We are going to have to keep going with this discussion. Perhaps they can continue afterward. One more very short question, please.

Dr Mohamed Emara (Cairo, Egypt). I congratulate you for this nice presentation. Did you try the end-systolic phase indexes rather than the ejection phase indexes? As we all know, in chronic volume overload, when a leak is present in the mitral valve, EF is usually higher than normal, so one might start with the wrong judge on the ventricle.

Dr Suri. Sorry, did we consider other modalities?
Dr Emara. No, other indexes to evaluate the left ventricle. You used the ejection phase indexes.

Dr Suri. It is a very good point. We have to remember 1 critical thing, and when we were writing the report we spoke about this several times. That is, although we might all use a varied and heterogeneous array of modalities to assess LV function in research studies, the reality is that echocardiographic assessment of LV systolic function using EF is ubiquitous in current clinical practice and deeply embedded in current clinical practice guidelines. Thus, although we can endeavor to explore different experimental means of assessing contractility and latent dysfunction, the truth is we did not examine in the present study in order to permit our findings to be generalized in the broader international clinical community. 